\title{
ACIDENTE VASCULAR CEREBRAL ISQUÊMICO EM UMA ENFERMARIA DE NEUROLOGIA: COMPLICAÇÕES E TEMPO DE INTERNAÇÃO
}

Rodrigo Bomeny de Paulo*1, Tales Mollica Guimarães², Paulo Victor Partezani Helito², Paulo Eurípides Marchiori³ ${ }^{3}$ Fabio luji Yamamoto 4 , Letícia Lessa Mansur ${ }^{5}$, Milberto ScafF ${ }^{6}$, Adriana Bastos Conforto ${ }^{7}$

Trabalho elaborado na Faculdade de Medicina da Universidade de São Paulo Hospital das Clínicas FMUSP, São Paulo, SP

*Correspondência:

Av. Divino Salvador, 286

apto 194

CEP 04078-013

São Paulo - SP

\section{RESUMO}

ОвлEтIvos. Os objetivos deste trabalho foram: avaliar as complicações e o tempo de internação de doentes com acidente vascular cerebral isquêmico ( $\mathrm{AVCl}$ ) na fase aguda ou subaguda em uma enfermaria de Neurologia geral em São Paulo; investigar a influência de idade, fatores de risco para doença vascular, território arterial acometido e etiologia sobre as complicações e o tempo de internação.

MÉtodos. Foram coletados prospectivamente dados de 191 doentes com AVCl e posteriormente analisados.

Resultados. Cinquenta e um doentes $(26,7 \%)$ apresentaram alguma complicação clínica durante a internação. A pneumonia foi a complicação mais frequente. 0 tempo médio de internação na enfermaria foi de $16,8 \pm 13,8$ dias. $\mathrm{Na}$ análise multivariável, o único fator que se correlacionou significativamente com menor taxa de complicações foi idade mais jovem ( $O R=0,92-0,97, p<0,001)$. O único fator que influenciou independentemente o tempo de internação foi a presença de complicações ( $O R=4,20$; $\mathrm{IC}=1,92-8,84 ; \mathrm{p}<0,0001)$.

Conclusã̃o. Estes resultados devem ser considerados no planejamento e organização do atendimento do AVCI no Brasil.

DescritoRes: Acidente cerebrovascular. Tempo de internação. Etiologia. Morbidade.

\section{INTRODUÇÃO}

Embora estime-se que dois terços das mortes por acidente vascular cerebral (AVC) ocorram em países em desenvolvimento, informações sobre epidemiologia, manejo e prognóstico nestes países são escassas ${ }^{1,2}$. Nos últimos anos, o AVC tem sido identificado como a primeira causa de morte no Brasil ${ }^{3,4}$. Em estudos brasileiros, o tipo mais frequente de AVC é o acidente vascular cerebral isquêmico $(\mathrm{AVCl})^{5,6}$. Complicações médicas intrahospitalares são comuns no AVCl e contribuem significativamente para a morbimortalidade desta doença. Foi estimado que $75 \%$ a 95\% dos doentes apresentem ao menos uma complicação após um AVCI, sendo que $24 \%$ desses pacientes apresentam alguma complicação que requer um tratamento imediato ou resulta em aumento do tempo de internação ou óbito ${ }^{7-8}$.

Os objetivos deste trabalho foram: analisar as complicações e o tempo de internação de doentes internados por AVCI na fase aguda ou subaguda em uma enfermaria de neurologia geral; investigar a influência de idade, fatores de risco para doença vascular, território arterial acometido e etiologia sobre as complicações e o tempo de internação.

\section{MÉtodos}

Foram coletados prospectivamente dados de 191 doentes com diagnóstico de AVCl internados na Enfermaria de Neurologia do Hospital das Clínicas da Universidade de São Paulo entre novembro de 2003 e novembro de 2005. Foram incluídos doentes maiores de 18 anos, com diagnóstico de AVCl comprovado por exame de neuroimagem (tomografia de crânio ou ressonância magnética de encéfalo) e início dos sintomas até 15 dias antes da admissão. Foram excluídos doentes reinternados para realização de exames complementares ou intervenções terapêuticas e início de sintomas há mais de 15 dias.

Todos os pacientes foram submetidos aos procedimentos de rotina para investigação e tratamento de $\mathrm{AVCl}$, de acordo

1. Aluno de pós-graduação do Hospital das Clínicas da Faculdade de Medicina da Universidade de São Paulo, São Paulo, SP

2. Aluno de graduação, Faculdade de Medicina da Universidade de São Paulo, São Paulo, SP

3. Professor Associado, Departamento de Neurologia da Universidade de São Paulo, São Paulo, SP

4. Médico assistente, Divisão de Clínica Neurológica do Hospital das Clínicas da Faculdade de Medicina da Universidade de São Paulo, São Paulo, SP

5. Professora Assistente do Departamento de Fisioterapia, Fonoaudiologia e Terapia Ocupacional da Faculdade de Medicina da Universidade de São Paulo, São Paulo, SP

6. Professor Titular, Departamento de Neurologia da Universidade de São Paulo, São Paulo, SP

7. Professora Colaboradora, Departamento de Neurologia da Universidade de São Paulo e médica assistente, Divisão de Clínica Neurológica do Hospital das Clínicas da Faculdade de Medicina da Universidade de São Paulo, São Paulo, SP 
com algoritmos padronizados pelo Grupo de Doenças Cerebrovasculares: avaliação clínica e neurológica, eletrocardiograma, hemograma, bioquímica, perfil lipídico, velocidade de hemossedimentação, sorologia para lues e doença de Chagas, ecocardiograma transtorácico, tomografia computadorizada ou ressonância magnética de encéfalo, duplex de artérias carótidas e vertebrais, angio-ressonância ou angio-tomografia intra e extracraniana. Doentes com idade menor ou igual a 45 anos, assim como doentes nos quais a etiologia do AVCI permaneceu indeterminada após a investigação básica, foram submetidos a avaliação reumatológica e hematológica, ecocardiograma transesofágico e, em casos selecionados, arteriografia por subtração digital, doppler transcraniano, Holter e exame de líquor. Foram analisados dados referentes a etiologia de acordo com critérios do estudo $\mathrm{TOAST}^{9}$, territórios arteriais acometidos (carotídeo, vértebro-basilar ou ambos), tempo de internação e complicações durante a internação. O estudo fez parte de um protocolo de Banco de Dados de AVC, aprovado pela Comissão de Ética para Análise de Projetos de Pesquisa da Diretoria Clínica do Hospital das Clínicas e da Faculdade de Medicina da Universidade de São Paulo.

Os aplicativos Minitab 13.0 e SPSS 10.0 foram utilizados para a análise estatística. O teste de Kolmogorov-Smirnov foi utilizado para verificar a normalidade dos dados. Na análise descritiva, médias e desvios padrão das médias foram utilizados para variáveis com distribuição normal, e medianas e intervalos em caso contrário. Testes de Qui quadrado foram utilizados para comparar etiologias de AVCls, e frequência de complicações em doentes com idade maior ou menor/igual a 45 anos. Os fatores idade, etiologia do $\mathrm{AVCl}$, território arterial acometido, fatores de risco, e a presença de complicações foram incluídos como variáveis independentes em um modelo de regressão multivariável, sendo a variável dependente o tempo de internação, dicotomizada em maior ou menor/igual que a média. Valores de $p<0,05$ foram considerados significantes. "Odds ratios" (OR) e intervalo de confiança (IC) foram apresentados para resultados significantes.

\section{Resultados}

A idade média dos doentes com AVCl admitidos na enfermaria de neurologia foi $53,4 \pm 16,4$ anos. A proporção de homens foi $62,3 \%$ e a proporção de doentes do grupo étnico branco, $77,5 \%$. As frequências de fatores de risco para doença vascular estão listadas na Tabela 1. 40,8\% dos pacientes apresentaram apenas um fator de risco, 26,2\%, dois fatores e 14,7\%, três ou mais. Vinte e três pacientes (12\%) apresentavam história prévia de AVC.

A Tabela 2 ilustra os territórios arteriais acometidos e a Tabela 3 as etiologias dos AVCls de acordo com critérios do estudo TOAST. Não encontramos diferenças significantes entre os territórios arteriais acometidos no grupo mais jovem (idade menor ou igual a 45 anos) e no grupo com mais de 45 anos $\left(c^{2}=1,56 ; p=0,21\right)$. Porém, houve uma diferença significante entre as causas de AVCI nas duas faixas etárias $\left(c^{2}=30,8 ; p\right.$ $<0,0001$ ): nos pacientes com idade igual ou maior que 45 anos, a etiologia mais frequente foi aterosclerose $(36,4 \%)$, enquanto que, nos mais jovens, a mais frequente foi indeterminada $(57,6 \%)$.

\begin{tabular}{|c|c|c|c|}
\hline \multicolumn{4}{|c|}{$\begin{array}{c}\text { Tabela1 - Fatores de risco - Principais fatores de risco para } \\
\text { doença vascular em doentes com idade igual a menor } \\
\text { a } 45 \text { anos, ou com mais de } 45 \text { anos. HAS, Hipertensão } \\
\text { arterial sistêmica.DM, diabetes mellitus }\end{array}$} \\
\hline Fatores de Risco & $n \leq 45 a(\%)$ & $n>45 a(\%)$ & $n(\%)$ \\
\hline HAS & $18(30,5)$ & $96(72,7)$ & $114(59,7)$ \\
\hline DM & $8(13,5)$ & $29(22,0)$ & $37(19,4)$ \\
\hline Obesidade & $7(11,9)$ & $17(12,9)$ & $24(12,6)$ \\
\hline Cardiopatias & $4 \quad(6,8)$ & $32(23,5)$ & $36(18,9)$ \\
\hline Dislipidemia & $8(13,5)$ & $32(24,2)$ & $40(21,0)$ \\
\hline Tabagismo & $31 \quad(52,5)$ & $83(62,9)$ & $114(59,7)$ \\
\hline
\end{tabular}

\begin{tabular}{lccc}
\hline \multicolumn{4}{l}{$\begin{array}{l}\text { Tabela } 2 \text { - Territórios arteriais. Territórios arteriais acometidos em } \\
\text { doentes com idade igual a menor a } 45 \text { anos, ou mais de } \mathbf{4 5} \text { anos }\end{array}$} \\
\hline & $\mathrm{n} \leq \mathbf{4 5 a}(\%)$ & $\mathrm{n}>\mathbf{4 5 a}(\%)$ & $\mathbf{N}(\%)$ \\
\hline Carotídeo & $40(67,8)$ & $83(62,9)$ & $123(64,4)$ \\
Vertebrobasilar & $15(25,4)$ & $39(29,6)$ & $54(28,3)$ \\
Carotídeo+vertebrobasilar & $4(6,8)$ & $10(7,5)$ & $14(7,3)$ \\
\hline
\end{tabular}

Tabela 3 - Etiologias. Classificação etiológica dos acidentes vasculares cerebrais isquêmicos, de acordo com critérios do estudo TOAST

\begin{tabular}{lccc}
\hline Mecanismos AVCI & $\mathbf{n} \leq \mathbf{4 5 a}(\%)$ & $\mathbf{n}>\mathbf{4 5}^{\mathrm{a}}(\%)$ & $\mathbf{( \% )}$ \\
\hline Aterosclerose & $3(5,1)$ & $48(36,4)$ & $51(26,7)$ \\
Embolia cardíaca & $12(20,3)$ & $33(25,0)$ & $45(26,6)$ \\
Indeterminado & $34(57,6)$ & $42(31,8)$ & $76(39,8)$ \\
Lacunar & $2(3,4)$ & $3(2,3)$ & $5(2,6)$ \\
\hline
\end{tabular}

Um total de $51(26,7 \%)$ pacientes apresentaram alguma complicação durante a internação. Aproximadamente $8 \%$ de todos os pacientes internados em nossa enfermaria apresentaram como complicação um quadro infeccioso pulmonar e cerca de 4\%, infecção urinária. A Tabela 4 ilustra as complicações apresentadas durante a internação. Pacientes com mais de 45 anos tiveram frequência de complicações $(33,4 \%)$ significativamente mais alta que pacientes mais jovens $\left(11,9 \% ; c^{2}=\right.$ $9,6 ; p=0,002)$. Na análise multivariável, o único fator que se associou a menor frequência de complicações foi a idade mais jovem $(O R=0,92-0,97, p<0,001)$. Etiologia, território arterial e número de fatores de risco não tiveram influência significante ( $p>0.05)$.

O tempo médio de internação na enfermaria foi de 16,8 $\pm 13,8$ dias. $29,6 \%$ dos pacientes ficaram internados por menos de 10 dias, $46,8 \%$ por 10 a 20 dias e $23,7 \%$ por mais de 20 dias. $\mathrm{Na}$ análise multivariável, o único fator que se correlacionou significativamente com o tempo de internação maior que 16 dias foi a presença de complicações $(O R=4,20 ; I C=1,92-8,84$; $p<0,0001$ ). 


\begin{tabular}{lccc}
\hline \multicolumn{4}{l}{ Tabela 4 - Complicações. Percentagens de complicações apresentadas durante a internação em relação ao número total de complicações } \\
\hline Complicações & $\mathbf{n} \leq \mathbf{4 5 a}$ & $\mathrm{n}>\mathbf{4 5 a}$ & $\mathbf{N}(\%)$ \\
\hline Pneumonia & 1 & 7 & $15(29,4)$ \\
Infecção de trato unitário & 0 & 4 & $7(13,9)$ \\
Febre de origem indeterminada & 2 & 2 & $6(11,7)$ \\
Trombose venosa profunda & 1 & 3 & $3(5,9)$ \\
Complicações de arteriografia/angioplastia & 2 & 14 & $(9,8)$ \\
Outras & 1 & $15(29,4)$ \\
\hline
\end{tabular}

Foram registrados cinco óbitos (2,6\%). Entre estes casos, três ficaram internados por mais de 20 dias e todos apresentaram alguma complicação durante a internação.

\section{Discussão}

O território arterial mais acometido em nosso estudo foi o território carotídeo (64,4\%), compatível com dados já presentes na literatura, como no serviço de Lausanne, no qual o território carotídeo foi acometido em $68 \%$ de 891 doentes com AVCI, e o vértebro-basilar em $26 \%^{10}$. Nos bancos de dados de Lausanne e o do New England Medical Center, a mortalidade na fase aguda de AVCIs no território vértebro-basilar não foi superior à mortalidade de AVCls em território carotídeo ${ }^{11}$. É possível que a causa, a gravidade, a localização e a extensão da lesão isquêmica sejam mais relevantes para a morbimortalidade no AVCI que o território arterial avaliado isoladamente. Não encontramos correlação significativa entre o território do $\mathrm{AVCl}$ com a duração da internação ou presença de complicações.

Nos pacientes jovens, a principal etiologia foi indeterminada, porém em proporção maior que as relatadas em outros estudos $(8 \%-26 \%)^{12,13}$. Em nosso serviço, nem todos os resultados de exames laboratoriais estão habitualmente disponíveis no momento da alta e é possível que a etiologia possa ter sido identificada posteriormente, no seguimento ambulatorial, em muitos doentes.

No atual estudo, assim como relatado na literatura, pacientes com mais de 45 anos apresentam complicações clínicas com maior frequência. Os idosos apresentam mais complicações por apresentarem geralmente AVCs mais graves e por serem mais susceptíveis a infecções ${ }^{14}$. A pneumonia e a infecção urinária foram as complicações mais frequentes, como descrito em outros estudos $^{14,15}$.Complicações clínicas, neurológicas e psiquiátricas interferem na recuperação e aumentam o custo do tratamento. Medidas de prevenção e reabilitação precoce podem diminuir o risco de complicações, diminuindo a morbimortalidade e melhorando o prognóstico após o $\mathrm{AVCl}^{16,17}$. Por exemplo, a avaliação sistemática da deglutição e a implementação de estratégias adequadas para alimentação podem contribuir para diminuir o risco de pneumonia ${ }^{18}$.

Em nosso estudo, a ocorrência de complicações clínicas apresentou uma correlação significante com a duração da internação. O tempo de internação em nossa enfermaria foi relativamente longo, comparado com o tempo de internação em unidades de $\mathrm{AVC}^{19}$. De acordo com a literatura, o tempo de internação está diretamente relacionado ao número de complicações, à gravidade do comprometimento neurológico, à idade e à organização do atendimento ${ }^{15,20}$.A duração da internação pode ter sido em parte atribuída a complicações, à morosidade na realização de exames de acordo com o algoritmo de investigação etiológica, em uma enfermaria geral de um hospital público.

A taxa de mortalidade após o AVCI é variável na literatura. O estudo de RANTTAS relata uma taxa de mortalidade em três meses de $14 \%$, sendo as complicações clínicas responsáveis por $51 \%$ desses óbitos ${ }^{14}$. No estudo NEMESIS, a taxa de mortalidade em 28 dias foi de $12 \%^{21}$. No presente estudo, a taxa de mortalidade na enfermaria foi significativamente inferior (2,6\%). Doentes mais jovens e com nível de consciência preservado na admissão (provavelmente com menor gravidade de comprometimento neurológico e maior estabilidade clínica) tenderam a ser admitidos com maior frequência. Portanto, muitos doentes graves que não tenham sobrevivido não foram incluídos em nossa casuística. Da mesma forma, doentes com infartos lacunares submetidos a investigação preliminar no pronto-socorro e com quadros mais leves representaram uma proporção muito pequena de nossa amostra.

Uma limitação de nosso estudo é a avaliação de doentes estáveis neurologicamente, internados em uma enfermaria, que não representam todos os doentes admitidos com $\mathrm{AVCl}$ em nosso serviço. Em nossa instituição, foi estimado que menos de $20 \%$ dos doentes com diagnóstico de AVC foram admitidos na enfermaria, em um período de três meses, em 200422. Estas características refletem o atendimento em uma enfermaria geral e não em uma unidade de AVC, unidade de terapia intensiva ou semi-intensiva. As especificidades da amostra limitam a comparação com outros estudos que incluíram amostras gerais de doentes com $\mathrm{AVCl}$ e, portanto, doentes com idades mais avançadas (como a maioria dos doentes com $\mathrm{AVCl}$, em diferentes regiões do mundo) e comprometimento neurológico grave ou muito leve. Este viés pode ter contribuído para a baixa taxa de complicações, quando comparada a amostras gerais de doentes com AVCl. Contudo, a amostra é representativa de uma população atendida em um serviço de neurologia geral em um hospital universitário de referência, em um grande centro urbano.

A maior taxa de complicações e o prolongamento da internação em doentes maiores de 45 anos, comparados a doentes mais jovens, e o tempo de internação relativamente longo em nosso serviço devem ser considerados no planejamento e organização do atendimento do AVCl. Providências que podem vir a ter grande impacto em saúde pública incluem organização do atendimento, otimização da realização de exames complementares, além de procedimentos simples como a prevenção de pneumonia aspirativa. Tais medidas têm o potencial de contribuir 
para a diminuição do tempo de internação, da morbidade e do impacto financeiro do $\mathrm{AVCl}$.

\section{SuPorte Financeiro}

Rodrigo Bomeny de Paulo recebeu bolsa de iniciação científica do Programa Institucional de Bolsas de Iniciação Cientifica do Conselho Nacional de Desenvolvimento Científico e Tecnológico (PIBIC/CNPq).

\section{Conflito de interesse: não há}

\section{SUMMARY}

Stroke in a NeURology WARD: etIologies, complications AND LENGTH OF STAY

InTRODUCTION. Purposes of this study were: evaluate complications and length of stay of patients admitted with diagnosis of ischemic stroke (IS) in the acute or subacute phase, in a general Neurology ward in São Paulo, Brazil; investigate the influence of age, risk factors for vascular disease, arterial territory and etiology.

Methods. Data from 191 IS patients were collected prospectively.

RESULTS. Fifty-one patients (26.7\%) presented at least one clinical complication during stay. Pneumonia was the most frequent complication. Mean length of stay was 16.8+-13.8 days. Multivariate analysis revealed a correlation between younger age and lower complication rates $(O R=0.92-0.97, p$ $<0.001)$. Presence of complications was the only factor that independently influenced length of stay $(O R=4.20 ; C l=1.92-$ 8.84; $p<0.0001$ ).

CONCLUSION. These results should be considered in the planning and organization of IS care in Brazil. [Rev Assoc Med Bras 2009; 55(3): 313-6]

KEY WORDS: Stroke. Length of stay. Etiology. Morbidity.

\section{REFERÊNCIAS}

1. Saposnik G, Del Brutto OH. Iberoamerican Society of Cerebrovascular Diseases. Stroke in South America: a systematic review of incidence, prevalence, and stroke subtypes. Stroke. 2003;34:2103-8.

2. Mathers CD, Lopez AD, Murray CJL. The burden of disease and mortality by condition: data, methods, and results for 2001. In: Lopez AD, Mathers CD, Ezzati M, Jamison DT, Murray CJL, editors. Global burden of disease and risk factors. New York: Oxford University Press; 2006. p.45-240.
3. Mansur AP, Souza MFM, Favarato D, Avakian SD, César LAM, Aldrigui JM, et al. Stroke and ischemic heart disease mortality trends in Brazil from 1979 to 1996. Neuroepidemiology. 2003;22:179-83.

4. Brasil. Ministério da Saúde. Sistema de informações sobre mortalidade. DATASUS - TABNET. Indicadores e dados básicos. Brasil, 2004. [citado 2007]. Disponível em: htttp://www.datasus.gov.br.

5. Cabral NL, Longo AL, Moro CH, Amaral CH, Kiss HC. Epidemiology of cerebrovascular disease in Joinville, Brazil. An institutional study. Arq Neuropsiquiatr. 1997;55(3A):357-63.

6. Minelli C, Fen LF, Minelli DPC. Stroke incidence, prognosis, 30-day and 1-year case fatality rates in Matão, Brazil. Stroke. 2007;38:2906-11.

7. Roth EJ, Lovell L, Harvey RL, Heinemann AW, Semik P, Diaz S. Incidence of and risk factors for medical complications during stroke rehabilitation. Stroke. 2001;32:523-9.

8. Langhorne P, Stott DJ, Robertson L, MacDonald J, Jones L, McAlpine C, et al. Medical complications after stroke: a multicenter study. Stroke. 2000;31:1223-9.

9. Adams HP, Bendixen BH, Kappelle J, Biller J, Love BB, Gorodon DL, et al. Classification of subtype of acute ischemic stroke. Definitions for use in a multicenter clinical trial. Stroke. 1993;24:35-41.

10. Bogousslavsky J, Van Melle G, Regli F. The Lausanne Stroke Registry: analysis of 1000 consecutive patients with first stroke. Stroke. 1998;19:1083-92.

11. Caplan LR. Posterior circulation disease: clinical findings, diagnosis and management. Cambridge: Blackwell Science; 1996. p.193-7.

12. Varona JF, Guerra JM, Bermejo F, Molina JA, Gomez de la Camara A. Causes of ischemic stroke in young adults, and evolution of the etiological diagnosis over the long term. Eur Neurol. 2007;57:212-8.

13. Varona JF, Bermejo F, Guerra JM, Molina JA. Long-term prognosis of ischemic stroke in young adults. Study of 272 cases. J Neurol. 2004;251:1507-14.

14. Johnston KC, Li JY, Lyden PD, Hanson SK, Feasby TE, Adams RJ, et al. Medical and neurological complications of ischemic stroke experience from the RANTTAS trial: RANTTAS investigators. Stroke. 1998;29:447-53.

15. Arboix A, Massons J, Oliveres M, Garcia L, Titus F. Mortalidad en la fase aguda de la enfermedad cerebrovascular: registro de La Alianza - Hospital Central de Barcelona. Med Clin (Barc). 1994;103:529-33.

16. McGuire JR, Harvey RL. The prevention and management of complications after stroke. Phys Med Rehabil Clin North Am. 1999;10:857-74.

17. Bae HJ, Yoon DS, Lee J, Kim BK, Koo JS, Kwon O. In-hospital medical complications and long-term mortality after ischemic stroke. Stroke. 2005;36:2441-5.

18. Foley N, Salter K, Teasell R. Specialized stroke services: a meta-analysis comparing three models of care. Cerebrovasc Dis. 2007;23:194-202.

19. Duncan PW, Zorowitz R, Bates B. Management of adult stroke rehabilitation care: a clinical practice guideline. Stroke. 2005;36:100-43.

20. Heuschmann PU, Kolominsky-Rabas PL, Misselwitz B, Hermanik P, Leffamann C, Von Reutern GM, et al. Factors influencing duration of hospitalization after stroke in Germany Dtsch Med Wochenschr. 2004;129:299-304.

21. Dewey HM, Sturm J, Donnan GA, Macdonnel RA, McNeil JJ, Thrift AG; North East Melbourne Stroke Incidence Study. Cerebrovasc Dis. 2003;15:133-9.

22. Conforto AB, Paulo RB, Patroclo CB, Pereira SLA, Miyahara HS, Fonseca CB, et al. Características demográficas, diagnóstico e tratamento emergencial de doentes com acidente vascular cerebral em um centro de referência universitário. Arq Neuropsiquiatr. 2007;65(Supl 2):20-1.

Artigo recebido: 20/02/08 Aceito para publicação: 14/01/09 